\title{
Tamaño poblacional y patrón de conducta de Grus canadensis (Aves: Gruidae) en dos localidades de Cuba
}

\author{
Yarelys Ferrer-Sánchez ${ }^{1,2}$, Idael Ruiz ${ }^{1}$, Dennis Denis ${ }^{3}$, Yordanis Torres ${ }^{3}$, \\ Fernando Abasolo-Pacheco ${ }^{2} \&$ Alexis H. Plasencia-Vázquez ${ }^{4}$ \\ 1. Empresa Nacional para la Protección de la Flora y la Fauna, $7^{\mathrm{ma}}$ No. 514, Playa, La Habana, Cuba; yferrersanchez@ \\ gmail.com, idaelruizc@gmail.com \\ 2. Facultad de Ciencias Ambientales y Ciencias Agrarias, Universidad Técnica Estatal de Quevedo, km 1 1/2 vía \\ Quevedo-Santo Domingo, Quevedo, Los Ríos, Ecuador; ferchoabasolo@gmail.com \\ 3. Universidad de la Habana, Calle 25, Plaza de la Revolución, La Habana, Cuba; dda@fbio.uh.cu \\ 4. Universidad Autónoma de Campeche, Campeche, México; alexpla79@gmail.com
}

$$
\text { Recibido 01-II-2016. Corregido 20-VI-2016. Aceptado 20-VII-2016. }
$$

\begin{abstract}
Population size and behavior pattern of Grus canadensis (Aves: Gruidae) in two localities of Cuba. The availability of information on species abundance in the Neotropic is insufficient, and this prevents the execution of precise analysis and the definition of adequate conservation strategies for endemic and threatened species. This study aimed to analyze the population size of the endemic and threatened subspecies Grus canadensis nesiotes. For this, a simultaneous census was undertaken in 24 count stations in Isla de la Juventud (IJ) and 32 stations in Ciego de Ávila (CA), Cuba, during two consecutive days between 2008 and 2010. Abundance and behavior pattern (instantaneous method) were analyzed by habitat type, to help understand how cranes modify their behavioral pattern when the natural habitat is changed. Flocks in IJ had three individuals, and between $1.9 \pm 1.5$ and $2.8 \pm 1.5$ in CA. Population size in IJ was 164 individuals, and in CA of 137,141 and 168 individuals for the 2008-2010 period, respectively. The counting efficacy was high (IJ: $91 \%$; CA: 81-87 \%) and the numerical concordance was intermediate (IJ: $45.4 \%$; CA: $72 \%$ ). When comparing the habitat type, the abundance was higher in natural savannahs (83), followed by coastal flats (59), pines (23) and cattle pastures (7) in IJ; while in CA, marsh grasslands hosted the greatest abundance for the three years period $(130 ; 120 ; 112)$, followed by grassland with palms $(2 ; 17 ; 51)$ and cattle pastures $(5 ; 4 ; 5)$. The cranes were fed more in cattle pastures and were more alert in natural savannas and marsh grasslands. The frequency of feeding and alert behaviors was different from the natural savannah/coastal flats and natural savannah/cattle pastures combinations in IJ. For $\mathrm{CA}$, differences were found between marsh grasslands and marsh grasslands with palms. The population size increased by management strategies adopted in CA; nevertheless, might be affected by habitat loss associated with invasive alien plants in IJ. We propose the maintenance of prescribed fire in marsh grasslands under protection regime, as a strategy for long-term management to contribute with population growth. Rev. Biol. Trop. 64 (4): 1495-1504. Epub 2016 December 01.
\end{abstract}

Key words: Cuban sandhill crane, abundance, endemic subspecies, threatened, habitat loss.

El conocimiento de la distribución y abundancia de una especie es decisivo para el diseño coherente de planes de conservación y manejo a escala de áreas, países y continentes (Myers, Mittermeier, Mittermeier, Fonseca, \& Kent, 2000). Este análisis remarca su importancia cuando se aplica en especies endémicas, raras, amenazadas o con hábitats restringidos, que generalmente no disponen de datos sobre sus preferencias de hábitats, historia natural y tendencias poblacionales. Actualmente, la disponibilidad de información sobre abundancia de especies es insuficiente en islas neotropicales como Cuba, lo que impide la realización de 
análisis precisos y la definición de estrategias de conservación adecuadas para el rescate de especies endémicas y amenazadas.

Las grullas son aves que necesitan de los humedales para su supervivencia, e incluyen en su distribución zonas modificadas cercanas a áreas húmedas (Nesbitt \& Williams, 1990). En Norteamérica, Grus canadensis es la más abundante y mejor distribuida. Entre las subespecies, Grus canadensis nesiotes (Gundlach, 1876) define el límite sur con las poblaciones más australes y aisladas genéticamente en el archipiélago cubano (Phymer, Fain, Austin, Johnson, \& Krajewski, 2001). Gundlach (1876) consideró a esta subespecie endémica como un ave común en algunas sabanas extensas del oeste y suroeste de Cuba y en Isla de la Juventud. Solo se reconocían cuatro poblaciones aisladas en Pinar del Río, Isla de la Juventud, Ciénaga de Zapata y Sabana de Lesca (Garrido \& García, 1975). Sin embargo, Gálvez y Chávez-Ramírez (2010) documentaron la existencia de 10 poblaciones con 526 individuos para Cuba. Este número crítico indica el grado de amenaza que presentan, provocado fundamentalmente por la pérdida y fragmentación de hábitats (Gálvez \& Ferrer, 2012).

Los ecosistemas donde habitan las grullas han sufrido sobrexplotación, procesos de drenaje artificial, y las consecuentes afectaciones en los regímenes hídricos. Esta pérdida contribuyó al descenso de las poblaciones de grullas por lo que solo cuatro (Isla de la Juventud, Ciénaga de Zapata, Sancti Spíritus y Ciego de Ávila) sobrepasan los 70 individuos (Gálvez \& Chávez-Ramírez, 2010). La población más numerosa habita en un ecosistema de arenas silíceas en Isla de la Juventud (Gálvez \& Chávez-Ramírez, 2010). No obstante, la segunda población se encuentra en el humedal norte de Ciego de Ávila. Las diferencias entre ecosistemas probablemente definen diferentes usos de hábitats y patrones de abundancia. La determinación de estos patrones puede proporcionar un conocimiento básico del estado de ambas poblaciones después de 10 años de monitoreo y manejo.
La modificación antropogénica es otro factor que influye en el uso de hábitat. Probablemente, el patrón de actividad de las grullas en cada hábitat pueda ser un indicador de la calidad y adecuación para la subespecie (Ferrer \& Ruiz, 2009). Estos estudios también pueden contribuir al análisis del estado de las poblaciones y a las medidas de manejo si se entiende cómo las grullas modifican su patrón conductual cuando el hábitat natural se modifica. Por tanto, el presente estudio evaluó el tamaño poblacional de $G$. c. nesiotes y caracterizó su patrón de actividad en un ecosistema de sabana seca y uno inundado de acuerdo a sus tipos de hábitats.

\section{MATERIAL Y MÉTODOS}

El estudio fue realizado en la región centro-noroeste de Isla de la Juventud (IJ; $21^{\circ} 46^{\prime} 24.89^{\prime \prime} \mathrm{N}-82^{\circ} 57^{\prime} 49.25^{\prime \prime} \mathrm{W}$ ) los días 19 y 20 de diciembre de 2008 y en el sitio Ramsar Gran Humedal del Norte de Ciego de Ávila (CA; 221'24.6” N - 78030'43.3” W), durante 1 y 2, 7 y 8,17 y 18 de febrero 2008, 2009 y 2010, respectivamente. El área de IJ incluyó la Reserva Ecológica Los Indios. Esta zona abarcó la cuenca hidrográfica Itabo-La Majagua-Los Indios, que se caracteriza fundamentalmente por la formación de sabanas de arenas de sílice. El área de CA abarcó la cuenca hidrográfica La Yana, con formaciones vegetales predominantes como los manglares, los bosques semideciduos mesófilos con humedad fluctuante, los bosques siempreverdes y los herbazales de ciénaga. También existen extensas áreas (antiguos herbazales) transformadas en pastizales y cultivos. En los herbazales de ciénaga y pastizales se ubicaron las estaciones de conteo.

Las dos áreas fueron divididas en subregiones para un mejor análisis de la distribución espacial de las grullas. En IJ se utilizaron seis de las ocho subregiones definidas por Gálvez, Berovides, Wiley y Rivera (1999): SiguaneaSan Pedro, Los Indios, Buena Vista, Esperanza-Demajagua, West Port-Sabana Grande y South-Central. Para CA se diferenciaron las 
subregiones: Venero, Loma de Cunagua, Laguna y Monte Malo, basado en los límites de áreas protegidas presentes y las características de escorrentía de la cuenca (Ferrer \& Ruiz, 2010).

Para determinar la abundancia (número de individuos) en los diferentes hábitats, se realizó un conteo simultáneo (Gálvez et al., 1999) en 32 estaciones de observación en CA y 24 en IJ que fueron ubicadas a partir de un diseño sistemático a cada $1.5-2 \mathrm{~km}$. Estas estaciones se ubicaron en hábitats con posibilidades de uso, clasificados por Gálvez, Berovides y Chávez-Ramírez (2005), y Ferrer y Ruiz (2010) (pastizal, sabana natural, pinar, marisma costera, herbazal de ciénaga, herbazal con palmas) y que coinciden con las mismas estaciones de conteos previos (Gálvez et al., 1999; Gálvez \& Chávez-Ramírez, 2010). Se usaron torres de observación de $10 \mathrm{~m}$ en las dos localidades, que abarcaban $\sim 0.81 \mathrm{~km}^{2}$ cada una. Estas torres estuvieron enmascaradas con la vegetación circundante para evitar el efecto de molestias por los observadores. Los observadores fueron capacitados para distinguir las siluetas de las grullas al vuelo y diferenciarlas de otras aves acuáticas.

Se contaron simultáneamente todas las grullas observadas o escuchadas (censo poblacional) entre 16:00-19:00 y 06:00-09:30 de dos días consecutivos (6h y 30 min por cada hábitat) una vez cada año (Gálvez et al., 1999). Se registró el número de grullas, hora de avistamiento, dirección de vuelo, tipo de hábitat y cobertura. Con esta información y las coordenadas geográficas de las estaciones de conteo, se eliminaron los conteos repetidos y se estimaron los siguientes parámetros: frecuencia de estaciones con conteos positivos, total de individuos contados, individuos/estación, individuos/bando, repetitividad de los conteos (IR) es igual al número de conteos consecutivos positivos más el número de conteos consecutivos negativos dividido por el total de estaciones de conteo y multiplicado por 100, y concordancia numérica (IC) es igual al número de conteos consecutivos con diferencia $\leq 5$ dividido por el total de estaciones con conteos positivos y multiplicado por 100 (indica en qué cuantía difieren la cantidad de aves observadas entre los dos días, en valores absolutos). Estos últimos índices dieron una idea de la aproximación del censo al tamaño poblacional real (Gálvez et al., 1999).

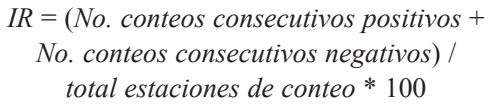

$I C=($ No. conteos consecutivos con diferencia $\leq 5 /$ total estaciones con conteos positivos) * 100

Durante los conteos de todos los años se realizó una descripción conductual en ambas localidades (592 observaciones) a través del método instantáneo (Altmann, 1974), teniendo en cuenta el análisis de Tacha, Vohs e Iverson (1987) para la selección del método de muestreo óptimo. Los registros se iniciaron al menos 30 min después de que las grullas fueron localizadas, con excepción de las que fueron escuchadas o vistas en vuelo. El registro de la conducta se hizo mediante un escaneo del grupo de grullas durante $10 \mathrm{~s}$. Para la identificación del comportamiento se siguieron las descripciones de Tacha et al. (1987) y Ferrer y Ruiz (2009). Cada comportamiento se clasificó dentro de las categorías: alimentación, locomoción, vuelo, canto, acicalamiento, interacción, alerta y descanso (Kreger, Estevez, Hatfield, \& Gee, 2004).

Para la determinación del tamaño poblacional se seleccionó el mayor valor de individuos del número total entre los dos horarios de conteo. Se estimó el error asociado con el algoritmo Jackknife. El tamaño y número de individuos/bandos para cada área fueron comparados entre años para CA a través de la prueba Kruskal-Wallis y con los resultados de IJ a través de la prueba U de Mann-Whitney. Se comparó el número de individuos/bando entre los horarios de conteo.

Se obtuvieron los porcentajes de estaciones con conteos positivos y negativos por área y hábitat, y se compararon con la prueba t para porcentajes en el software Stat Xact (Cytel, Massachusetts). La abundancia por tipo de hábitat se comparó a través de un análisis de 
CUADRO 1

Tamaño de los grupos de Grus canadensis nesiotes por horarios de conteo entre 2008-2010

TABLE 1

Size of groups of Grus canadensis nesiotes by count schedules during 2008-2010

\begin{tabular}{|c|c|c|c|c|c|c|}
\hline \multirow{2}{*}{ Localidad/Año } & & \multicolumn{2}{|c|}{ Mañana } & \multicolumn{2}{|c|}{ Tarde } & \multirow{2}{*}{$\begin{array}{l}\text { Número total de } \\
\text { individuos }\end{array}$} \\
\hline & & Avistamientos & Tamaño de grupos & Avistamientos & Tamaño de grupos & \\
\hline Isla de la Juventud & 2008 & 70 & $3.2(1.8)$ & 73 & $3.1(2.0)$ & 164 \\
\hline \multirow[t]{3}{*}{ Ciego de Ávila } & 2008 & 45 & $2.1(1.5)$ & 73 & $2.2(1.2)$ & 137 \\
\hline & 2009 & 48 & $2.1(1.2)$ & 59 & $2.0(1.5)$ & 141 \\
\hline & 2010 & 52 & $2.8(1.5)$ & 58 & $1.9(1.5)$ & 168 \\
\hline
\end{tabular}

Media (desviación estándar).

varianza por permutaciones (10000 permutaciones) en el software PERMANOVA (Anderson, 2001). La frecuencia de las conductas alimentación y alerta se compararon entre hábitats con la Probabilidad exacta de Fisher.

\section{RESULTADOS}

El número de grullas por bando para IJ fue de tres individuos, resultado que coincidió entre los dos horarios de conteo. En CA la diferencia más notable se encontró en 2010, observándose grupos mayores $(2.8 \pm 1.5)$ en la mañana (Cuadro 1). El total de grullas/horario en IJ fue mayor durante la tarde (161 individuos), mientras que en la mañana se obtuvo el $83.2 \%$ (134 individuos). Las mayores variaciones en CA se obtuvieron en la tarde del 2008 (136), con un $34.8 \%$ superior a las 101 grullas contadas en la mañana siguiente. El tamaño de los bandos/horario no fue diferente entre años en $\mathrm{CA}(\mathrm{U}=28793 ; \mathrm{P}=0.7)$, pero si difirieron con $\mathrm{IJ}(\mathrm{U}=2$ 622.5; $\mathrm{P}=0.007)$.

El tamaño de la población en IJ fue de $163.8 \pm 1.3$ individuos. En CA, para 2008, se obtuvieron $136.5 \pm 1.3$ individuos. Durante 2009 y 2010 aumentó el tamaño poblacional a $140.6 \pm 0.95$ y $168 \pm 1.1$ individuos respectivamente, sin diferencias entre años $[\mathrm{H}(2,73)=$ $2.45 ; \mathrm{P}=0.3$ ], pero si entre las áreas de estudio $(\mathrm{U}=549 ; \mathrm{P}=0.04)$.

Las estaciones con conteos positivos superaron el $50 \%$. En CA el mayor porcentaje se obtuvo en 2010 (72\%); muy similar al $75 \%$ obtenido en IJ. El resto de los años tuvieron valores más bajos (2008: 53 \% y 2009: 66 $\%)$. Las diferencias entre años fueron significativas $(P=0.03)$, pero no entre los tipos de hábitat $(\mathrm{P}=0.14)$.

El porcentaje de estaciones con conteos positivos en el herbazal de ciénaga de CA fue 100,77 y $80 \%$ para los tres años, respectivamente. En el pastizal se obtuvieron los menores porcentajes durante 2008 y 2009. En IJ la sabana natural tuvo conteos positivos en 85.7 $\%$ de las estaciones y la marisma costera, el pastizal y el pinar tuvieron $100 \%$. La eficacia obtenida resultó mayor para IJ (91\%), aunque los valores para CA también fueron altos (81$87 \%)$. El mayor IC se obtuvo en 2009 para CA (72\%), mientras que en IJ estuvo entre los menores (45.4\%).

El número de grullas difirió entre intervalos de 15 min. Durante la mañana, fue mayor en los primeros minutos del conteo (Fig. 1A). En la tarde, el mayor número se observó después de las 18:00 horas, con variaciones posteriores. A inicios del conteo vespertino en CA (resumen de los tres años de conteo), se obtuvo un alto número de grullas, que disminuyó con el avance del tiempo y tuvo aumentos sobre las 18:00 horas (Fig. 1B).

La abundancia de grullas fue mayor en las sabanas naturales (83), seguido de las marismas costeras (59), pinares (23) y pastizales (7) en IJ. En CA los herbazales de ciénaga albergaron la mayor abundancia en los tres años (130; $120 ; 112)$, seguido del herbazal con palmas 


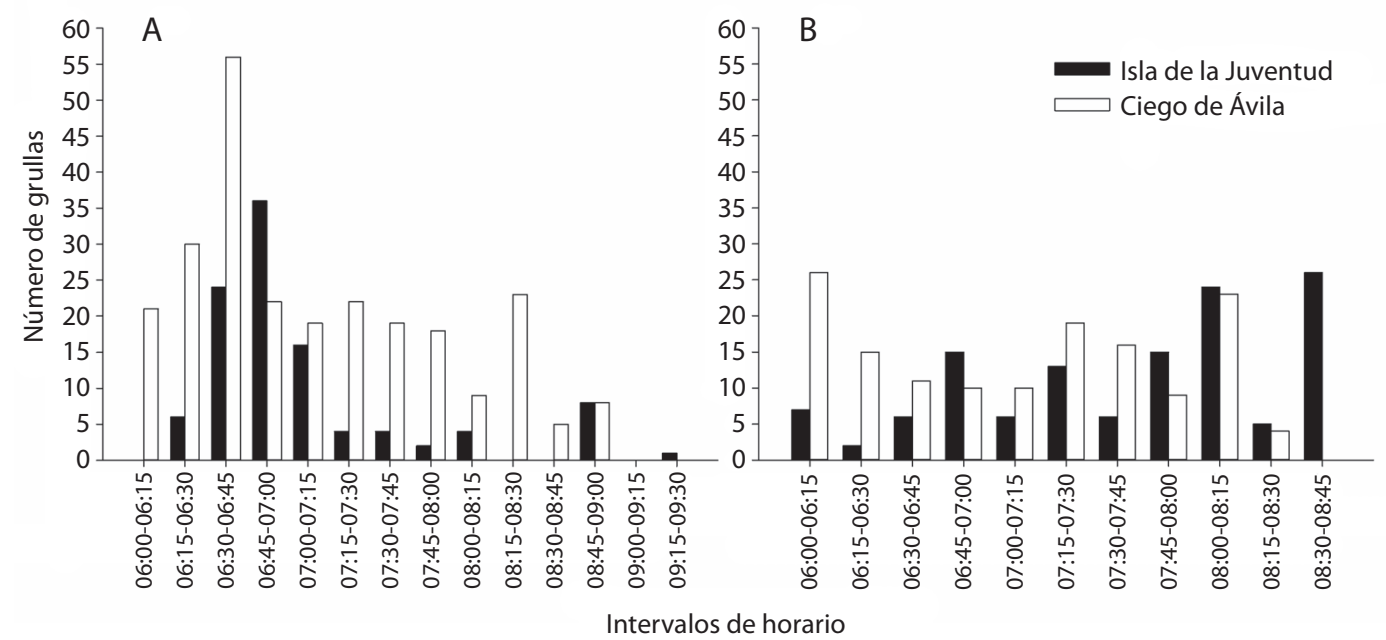

Fig. 1. Número de grullas (Grus canadensis nesiotes) por intervalo de tiempo, durante el conteo en Isla de la Juventud y Ciego de Ávila, Cuba. A: matutino; B: vespertino.

Fig. 1. Number of sandhill cranes (Grus canadensis nesiotes) per time interval, during counting in Isla de la Juventud and Ciego de Ávila, Cuba. A: morning; B: afternoon.

$(2 ; 17 ; 51)$ y los pastizales $(5 ; 4 ; 5) . \mathrm{La}$ abundancia fue diferente entre Pinar/SabanaPastizal $\left(\mathrm{P} \_\right.$permanova $=0.003 ; \mathrm{P} \_$MonteCarlo $=0.001)$ y Herbazal-Pastizal $\left(\mathrm{P} \_\right.$permanova $=$ 0.04; P_MonteCarlo $=0.04)$. Las combinaciones Pinar/Sabana-Herbazal con palma, Pinar/ Sabana-Marisma, Pastizal-Marisma y PastizalSabana natural tuvieron diferencias solamente con una prueba estadística.

El porcentaje de conductas fue similar en IJ (Fig. 2). Solamente la alimentación fue mayor en el horario de la tarde (29\%) respecto a la mañana $(17 \%)$, y lo contrario ocurrió con el acicalamiento, registrándose en mayor proporción durante la mañana $(8 \%)$. La alerta fue alta para ambos horarios $(21 \%$ tarde, $24 \%$ mañana) y no se registraron evidencias de descanso e interacción. Para CA hubo mayores diferencias entre horarios. Sin embargo, se mantuvo similar el porcentaje de alertas en 2008. La alerta fue más frecuente durante la mañana en los conteos de 2009 y 2010 (21\%,

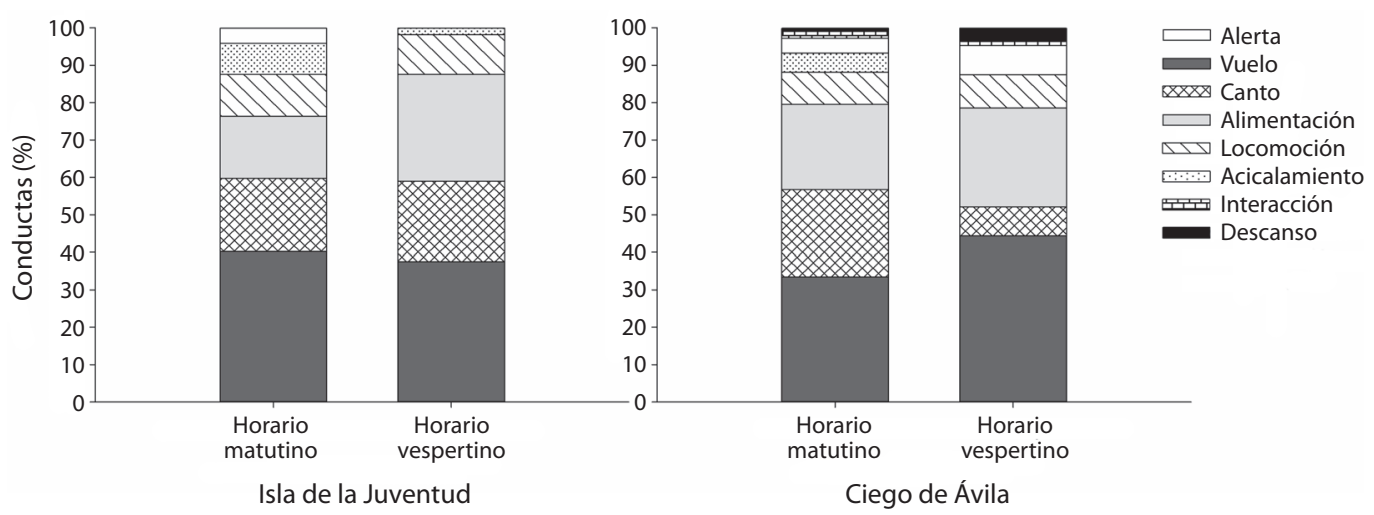

Fig. 2. Conductas de Grus canadensis nesiotes observadas durante los conteos de 2008-2010 en Isla de la Juventud y Ciego de Ávila, Cuba.

Fig. 2. Grus canadensis nesiotes behaviors observed during the 2008-2010 counts in Isla de la Juventud and Ciego de Ávila, Cuba. 
$45 \%)$, mientras que el vuelo lo fue durante la tarde $(67 \%, 39 \%)$.

La alerta fue proporcionalmente mayor en las sabanas naturales (38\%) de IJ respecto al resto de los hábitats $(9 \%$-pastizal y pinar, $14 \%$-marisma costera), y la alimentación fue mayor en los pastizales (41\%) con valores más bajos en pinar $(11 \%)$, marisma $(23 \%)$ y sabana natural (24\%). En las marismas costeras la alimentación, locomoción, alerta, vuelo $\mathrm{y}$ acicalamiento estuvieron en proporciones aproximadamente similares $(23 \%, 21 \%, 14 \%$, $27 \%, 15 \%$ respectivamente) y la locomoción fue mayor respecto al resto de los hábitats (4 $\%$-pinar, $5 \%$-sabana natural, $9 \%$-pastizal).

En CA, durante 2008, los hábitats utilizados fueron los pastizales y los herbazales de ciénaga. En los pastizales se observó la alimentación $(80 \%)$ y el vuelo $(20 \%)$ y la primera conducta fue 2.4 veces mayor respecto a los herbazales de ciénaga (33\%). Sin embargo, en los herbazales se registró mayor diversidad conductual (32\%-vuelo, $28 \%$-alerta, $4 \%$-acicalamiento, $2 \%$-descanso, $1 \%$-locomoción). Durante 2009 se observó en mayor proporción el vuelo en el herbazal respecto al herbazal con palmas ( $54 \%, 42 \%$ respectivamente), mientras que la alerta fue 2.4 veces mayor en el segundo hábitat $(27 \%)$. Para el herbazal de ciénaga se registraron conductas menos frecuentes como la interacción (3\%) y el descanso (3\%). La alimentación se mantuvo en porcentajes cercanos (19\%-herbazal y $27 \%$-herbazal con palmas). Para 2010, la alerta fue mayor en herbazales con palmas $(67 \%)$ respecto al herbazal (16 $\%)$ y pastizal (0), mientras que el vuelo fue mayor en pastizales (86\%). En el herbazal se registraron todas las conductas y entre estas la alimentación fue mayor respecto al resto de los hábitats (27\%-herbazal, $14 \%$-pastizal, 9 $\%$-herbazal con palmas).

La frecuencia de alimentación y alerta tuvo variaciones en los diferentes tipos de hábitat. Sin embargo, fueron significativas en la combinación sabana natural-marisma (P de Fisher $\left.=0.05 ; \mathrm{x}^{2}=4.3 ; \mathrm{P}=0.04\right)$ y sabana naturalpastizal $\left(\mathrm{P}\right.$ de Fisher $=0.02 ; \mathrm{x}^{2}=6.9 ; \mathrm{P}=0.01$ ) en IJ. Para CA las diferencias fueron entre herbazal-herbazal con palmas $(\mathrm{P}$ de Fisher $=$ $\left.0.001 ; \mathrm{x}^{2}=11.6 ; \mathrm{P}=0.001\right)$.

\section{DISCUSIÓN}

La abundancia de grullas fue mayor que todos los conteos previos que han utilizado la misma metodología en el sector de Ciego de Ávila. Sin embargo, en Isla de la Juventud fue mayor al referido por Gálvez et al. (1999) para 1995 y relativamente bajo respecto a 1998 (alrededor de 115 y 171 individuos respectivamente; Gálvez \& Chávez-Ramírez, 2010). La diferencia respecto a 1995 puede ser resultado de una mayor precisión en los conteos del 2010, en los que se emplearon dos días y dos horarios de conteo. Respecto a 1998 hubo un ligero decremento que puede ser indicio de presiones por la pérdida del hábitat de alimentación asociado a la expansión de la planta exótica Dichrostachys cinerea en pastizales y sabanas naturales (Muñoz, Pereda, Ponce, Cruz, \& Olazábal, 2009). La pérdida de hábitat conlleva al desplazamiento de individuos hacia zonas alejadas de la protección del área protegida Reserva Ecológica Los Indios y sus zonas aledañas (Urbanek, Szyszkoski, \& Zimorski, 2014) donde pueden enfrentar amenazas como la cacería furtiva, que conduce rápidamente a un decline en poblaciones pequeñas. Este tema se debe considerar con intensidad para trazar estrategias de manejo de hábitat y conservación de la población.

En CA el tamaño poblacional aumentó respecto al 2002 (102 grullas; Gálvez \& ChávezRamírez, 2010). Este aumento probablemente sea una respuesta a las acciones de manejo dentro del área protegida Refugio de Fauna El Venero iniciadas en 2004 (e.g. campaña educativa para las comunidades del humedal, plan de monitoreo de la población y manejo de hábitat). Una de las estrategias seguidas dentro del manejo de hábitat fue el uso de un régimen de fuego controlado y sistematizado, para propiciar hábitats de alimentación dentro de los herbazales de ciénaga protegidos. El fuego contribuye a la simplificación estructural de la vegetación herbácea y de una forma 
indirecta, se eliminan depredadores de polluelos y huevos (Bennett, 1989). Las grullas se alimentan dentro de estas zonas quemadas con mayor frecuencia que áreas sin quemar y acuden inmediatamente después de las quemas. De forma similar ocurre con G. americana en Aransas y se sugiere que también podrían usar las áreas quemadas para obtener minerales y como sitios de descanso (Chávez-Ramírez, Hunt, Slack, \& Stehn, 1996). Por otra parte, el crecimiento posterior de la vegetación garantiza sitios de anidación adecuados en herbazales de ciénaga para la grulla cubana (Ferrer, Denis, \& Ruiz, 2010). Por los beneficios que las áreas quemadas brindan a las grullas (Bishop, 1984; Chávez-Ramírez et al., 1996) y teniendo en cuenta el aumento poblacional de estas aves en $\mathrm{CA}$, se propone como estrategia de manejo a largo plazo el mantenimiento de las quemas controladas, que ya se realizan en unidades definidas de herbazales de ciénaga bajo régimen de protección.

Otra de las acciones realizadas fue la extracción del ganado de las áreas de anidación. Se conoce que en humedales de México, la actividad ganadera de los sistemas tradicionales de libre pastoreo manifiesta una amenaza que repercute en la conservación de los hábitats de las grullas (Clemente-Sánchez, Cortez-Romero, Palacio-Núñez, \& Rosas-Rosas, 2014). No es el sistema de pastoreo empleado, sino el grado de uso que se le da al hábitat por el tiempo que pastorea el ganado, y el número de cabezas por unidad de superficie. Esto reduce la capacidad de carga del hábitat, lo que degrada y reduce la producción de todos los animales que lo requieren (Holechek, Pieper, \& Herbel, 1989).

Las grullas tienen un potencial reproductivo bajo (Johnson \& Kendall, 1997), por ello se considera relevante el incremento de la población en un período de siete años. El incremento aunado a la protección, podría garantizar su supervivencia, si se mitigan las amenazas y si no existen problemas genéticos característicos de poblaciones pequeñas y aisladas como todas las poblaciones de grullas en Cuba (Gálvez \& Chávez-Ramírez, 2010). La reducción y fragmentación del hábitat podría contribuir al decline poblacional en todas las áreas ya que todas las poblaciones están separadas por distancias mayores al movimiento de dispersión más largo que se ha registrado para grullas no migratorias (48.3 km en Florida; Nesbitt, Schwikert, \& Folk, 2002).

El tamaño poblacional de G. c. nesiotes en IJ es aproximadamente igual a CA. Sin embargo, las características de los hábitats son diferentes lo que puede estar determinando también las diferencias entre el tamaño de los bandos. La riqueza vegetal y animal de las sabanas de sílice, así como la estructura de la vegetación han aportado un hábitat adecuado y diferente de lo que se conoce para la especie en Norteamérica (Krapu, Facey, Fritzel, \& Johnson, 1984; Tacha, Nesbitt, \& Vohs, 1992; López-Saut, Chávez-Ramírez, \& RodríguezEstrella, 2011). Es probable que esta población presente un nicho especializado y anidado dentro del nicho ecológico de G. canadensis, diferente también al resto de las ocho poblaciones de grullas en Cuba. Además, en IJ las fechas del conteo en diciembre estuvieron más aisladas temporalmente de la etapa reproductiva (marzo-junio; Gálvez et al., 2005) que las fechas de conteo en CA (febrero). Por lo tanto, en IJ se podían observar con mayor frecuencia grupos familiares de tres grullas (pareja y cría joven), mientras que en CA las parejas se estaban separando de los jóvenes para iniciar una nueva etapa de anidación.

La alta eficacia de los conteos da idea de la aproximación del resultado a la población real y a los hábitats que utiliza la subespecie durante la época no reproductiva. La mayor concentración de grullas se dispone hacia la zona centro-oeste de la Cuenca ItaboLa Majagua-Los Indios. En la Cuenca La Yana ocurre algo similar, al agruparse en la zona de concentración hídrica, donde confluyen los canales y manantiales.

En IJ existe mayor diversidad de hábitats utilizados por las grullas respecto a CA. En ambas áreas los hábitats naturales concentran la mayor cantidad de aves, proporcionan fuentes de alimento y permiten buena visibilidad ante la 
llegada de depredadores y disturbios (Gálvez et al., 2005; Ferrer et al., 2010). A pesar del poco disturbio en sabanas naturales, las grullas se mantienen alertas con mayor frecuencia respecto a los pastizales donde se alimentan más. Este comportamiento se relaciona con la estructura de la vegetación de las sabanas donde existen arbustos que contribuyen al buen camuflaje de los nidos. Sin embargo, fuera de la etapa reproductiva, las grullas se mantienen en vigilia con mayor frecuencia en este sitio. Por el contrario, los pastizales son extensos y abiertos, y a pesar de que el disturbio es mayor, las aves se alimentan más de lo que vigilan pues tienen suficiente distancia y visibilidad para detectar posibles amenazas (Treves, 2000).

En CA ocurre lo contrario, las grullas se alimentan con mayor frecuencia en herbazales de ciénaga abiertos y realizan una variedad mayor de conductas que reflejan la calidad de estos sitios para el descanso, acicalamiento y posteriormente la anidación. El uso de pastizales podría ser una consecuencia de la reducción de la capacidad de carga de los humedales naturales cuando existe sequía intensa, que provoca un desplazamiento de las aves para encontrar alimento extra y cubrir los requerimientos energéticos (e.g. Czech \& Parsons, 2002; Wood et al., 2010). Asimismo, estos pastizales son quemados frecuentemente por los campesinos y las grullas acuden inmediatamente después de las quemas para alimentarse de insectos, lagartos y anfibios muertos (Ruiz, com. pers.). A pesar de esto, los pastizales tienen disturbio humano que puede ser visto como un riesgo de depredación que afecta la conducta y supervivencia de los animales que estén forrajeando (Frid \& Dill, 2002).

A diferencia de lo observado en IJ, $G$. monacha dedica más tiempo a la vigilancia en los arrozales perturbados que en humedales naturales de la Reserva Natural Lago Shengjin en China (Li, Zhou, Xu, Zhao, \& Beauchamp, 2015). Sin embargo, también se alimentan más a expensas de conductas como el acicalamiento y la locomoción. Este comportamiento es consistente con resultados previos de G. c. nesiotes en $\mathrm{CA}$, donde se refiere que las grullas en los herbazales de ciénaga más perturbados se mantienen más tiempo en alerta, pero también se alimentan más y en menor tiempo por la mayor diversidad de alimentos (Ferrer \& Ruiz, 2009). En consecuencia, las grullas enfrentan una elección entre el forrajeo en lugares ricos en alimentos pero con riesgo de ser perturbadas con mayor frecuencia, y entre el forrajeo en lugares con menor riqueza y abundancia de alimentos pero menor frecuencia de disturbios.

Estos resultados constituyen una fuente de información importante para la evaluación de la tendencia poblacional y estado de los hábitats. Además, contribuyen al manejo y conservación de la subespecie. Es importante resaltar que la evaluación del tamaño poblacional, así como la presencia de grullas en los humedales, podría informar indirectamente sobre la salud de los hábitats ya que esta especie puede ser usada como indicadora de la composición y riqueza de aves acuáticas en los humedales (López-Saut, Rodríguez-Estrella, \& ChávezRamírez, 2014).

\section{AGRADECIMIENTOS}

Agradecemos al personal que participó en los conteos y a la Empresa Nacional para la Protección de la Flora y la Fauna por el apoyo logístico. A la ONG Children are the Hope por el financiamiento de los festivales. A IdeaWild y Birders' Exchange por los equipos donados y a los revisores por sus comentarios.

\section{RESUMEN}

La disponibilidad de información sobre abundancia de especies en el Neotrópico es insuficiente. Esto impide la realización de análisis precisos y definición de estrategias de conservación adecuadas para especies endémicas y amenazadas. A través de un censo simultáneo durante dos días consecutivos en 24 estaciones de conteo en Isla de la Juventud (IJ) y 32 estaciones en Ciego de Ávila (CA), Cuba, se obtuvo el tamaño poblacional de la subespecie endémica y amenazada Grus canadensis nesiotes durante 2008-2010. Se analizó la abundancia y patrón conductual (método instantáneo) por hábitat, para ayudar a entender cómo las grullas modifican su patrón conductual cuando el hábitat natural se modifica. Los bandos de grullas tuvieron tres individuos en IJ y entre $1.9 \pm 1.5$ y $2.8 \pm 1.5$ en CA. 
El tamaño poblacional en IJ fue de 164 individuos y en CA fueron 137, 141 y 168 individuos para 2008-2010. La eficacia del conteo fue alta (IJ: $91 \%$; CA: $81-87 \%$ ) y la concordancia numérica fue intermedia (IJ: $45.4 \%$; CA: 72 $\%)$. La abundancia fue mayor en sabanas naturales (83), seguido de marismas (59), pinares (23) y pastizales (7) en IJ. En CA los herbazales de ciénaga albergaron la mayor abundancia en los tres años $(130 ; 120 ; 112)$, seguido del herbazal con palmas $(2 ; 17 ; 51)$ y los pastizales $(5 ; 4 ; 5)$. Las grullas se alimentaron más en los pastizales y estuvieron más alerta en la sabana natural y el herbazal de ciénaga. La frecuencia de las conductas alimentación y alerta fueron diferentes entre las combinaciones sabana natural/marisma y sabana natural/pastizal en IJ. Para CA las diferencias fueron entre herbazal/herbazal con palmas. El tamaño poblacional aumentó en CA por estrategias de manejo adoptadas, pero en IJ puede afectarse por la pérdida de hábitat asociado a la invasión de plantas exóticas. Se propone como estrategia de manejo a largo plazo el mantenimiento de las quemas controladas en herbazales de ciénaga bajo régimen de protección para contribuir al aumento poblacional.

Palabras clave: grulla cubana, abundancia, subespecie endémica, amenazada, pérdida de hábitat.

\section{REFERENCIAS}

Altmann, J. (1974). Observational study of behavior: Sampling methods. Behavior, 49, 227-267.

Anderson, M. J. (2001). A new method for non-parametric multivariate analysis of variance. Austral Ecology, 26, 32-46.

Bennett, A. J. (1989). Movements and home ranges of Florida Sandhill Crane. Journal of Wildlife Management, $53,830-836$.

Bishop, M. A. (1984). The dynamics of subadult flocks of whooping cranes wintering in Texas, 1978-79 through 1982-83 (M.S. thesis). Texas A\&M University, College Station.

Chávez-Ramírez, F., Hunt, H. E., Slack, R. D., \& Stehn, T. V. (1996). Ecological correlates of whooping crane use of fire-treated upland habitats. Conservation Biology, 10, 217-223.

Clemente-Sánchez, F., Cortez-Romero, C., Palacio-Núñez, J., \& Rosas-Rosas, O. (2014). Estado de conservación del hábitat para Grulla Gris (Grus canadensis) $\mathrm{y}$ anátidos asociados en humedales de Zacatecas. AGROProductividad, 7, 49-56.

Czech, H. A., \& Parsons, K. C. (2002). Agricultural wetlands and waterbirds: a review. Waterbirds, 25, 56-65.

Ferrer, Y., \& Ruiz, I. (2009). Patrón de actividad diurna de la Grulla Cubana (Grus canadensis nesiotes
Gundlach, 1875) en el Refugio de Fauna El Venero, Cuba. Mesoamericana, 13, 6-11.

Ferrer, Y., \& Ruiz, I. (2010). Caracterización de los parámetros reproductivos de Grus canadensis nesiotes (Aves: Gruidae) en los herbazales de ciénaga del norte de Ciego de Ávila, Cuba. Mesoamericana, 14, 7-16.

Ferrer, Y., Denis, D., \& Ruiz, I. (2010). Caracterización y selección del sitio de anidación de la Grulla Cubana (Grus canadensis nesiotes) en el herbazal del Refugio de Fauna El Venero, Cuba. Animal Biodiversity and Conservation, 33, 19-29.

Frid, A., \& Dill, L. M. (2002). Human-caused disturbance stimuli as a form of predation risk. Conservation Ecology, 6, 11-26.

Gálvez, X., \& Chávez-Ramírez, F. (2010). Distribution, abundance, and status of Cuban sandhill cranes (Grus canadensis nesiotes). The Wilson Journal of Ornitho$\log y, 122,556-562$.

Gálvez, X., \& Ferrer, Y. (2012). Grus canadensis nesiotes. En H. González, L. Rodríguez, A. Rodríguez, C. A. Mancina, \& I. Ramos (Eds.), Libro Rojo de los Vertebrados de Cuba (pp. 219-220). La Habana: Editorial Academia.

Gálvez, X., Berovides, V., Wiley, J. W., \& Rivera, J. (1999). Population size of Cuban parrots Amazona leucocephala and sandhill cranes Grus canadensis and community involvement in their conservation in northern Isla de la Juventud, Cuba. Bird Conservation International, 9, 97-112.

Gálvez, X., Berovides, V., \& Chávez-Ramírez, F. (2005). Nesting ecology and productivity of the Cuban sandhill crane on the Isle of Youth, Cuba. Proceeding of North American Crane Workshop, 9, 225-236.

Garrido, O. H., \& García, F. (1975). Catálogo de las aves de Cuba. La Habana: Academia de Ciencias.

Gundlach, J. (1876). Contribución a la ornitología cubana. La Habana: La Antilla.

Holechek, J. L., Pieper, R. D., \& Herbel, C. H. (1989). Range management principles and practices. New Jersey: Prentice Hall.

Johnson, D. H., \& Kendall, W. L. (1997). Modeling the population dynamics of Gulf Coast sandhill cranes. Proceeding of the North American Crane Workshop, $7,173-180$

Krapu, G. L., Facey, D. E., Fritzel, E. K., \& Johnson, D. H. (1984). Habitat use by migrant sandhill crane in Nebraska. Journal of Wildlife Management, 48, 407-417.

Kreger, M. D., Estevez, I., Hatfield, J. S., \& Gee, G. F. (2004). Effects of rearing treatment on the behavior of 
captive whooping cranes (Grus americana). Applied Animal Behavior Science, 89, 243-261.

Li, C., Zhou, L., Xu, L., Zhao, N., \& Beauchamp, G. (2015). Vigilance and activity time-budget adjustments of wintering hooded cranes, Grus monacha, in human-dominated foraging habitats. PLOS ONE, 10: e0118928. doi:10.1371/journal.pone.0118928.

López-Saut, E. G., Chávez-Ramírez, F., \& RodríguezEstrella, R. (2011). New records of wintering grounds for sandhill cranes in Mexico. Waterbirds, 34, 239-246.

López-Saut, E. G., Rodríguez-Estrella, R., \& ChávezRamírez, F. (2014). ¿Son las grullas indicadoras de la riqueza de especies de aves acuáticas en humedales en el Altiplano Mexicano? Acta Zoológica Mexicana, 30, 268-287.

Muñoz, D., Pereda, J., Ponce, M., Cruz, Madelín, \& Olazábal, M. (2009). Recuperación de áreas de pastizales infestadas de Dichrostachys cinerea (marabú) combinando chapea mecanizada y pastoreo ovino. Ciencia y Tecnología Ganadera, 3(3), 139-142.

Myers, N., Mittermeier, R. A., Mittermeier, C. G., Fonseca, G. A. B., \& Kent, J. (2000). Biodiversity hotspots for conservation priorities. Nature, 403, 853-858.

Nesbitt, S. A., \& Williams, K. S. (1990). Home range and habitat use of Florida sandhill cranes. Journal of Wildlife Management, 54, 92-96.
Nesbitt, S. A., Schwikert, S. T., \& Folk, M. J. (2002). Natal dispersal in Florida sandhill cranes. The Journal of Wildlife Management, 66, 349-352.

Phymer, J. M., Fain, M. G., Austin, J. E., Johnson, D. H., \& Krajewski, C. (2001). Mitochondrial phylogeography, subspecific taxonomy, and conservation genetics of sandhill cranes (Grus canadensis; Aves: Gruidae). Conservation Genetics, 2, 203-218.

Tacha, T. C., Vohs, P. A., \& Iverson, G. (1987). Time and energy budget of sandhill cranes from midcontinental North America. Journal of Wildlife Management, $51,440-448$.

Tacha, T. C., Nesbitt, S. A., \& Vohs, P. A. (1992). Sandhill Crane. En A. Poole, P. Stettenheim, \& F. Gill (Eds.), The Birds of North America, (No. 31, pp. 1-24). Washington, D.C., Philadelphia, Pennsylvania: American Ornithologists' Union, The Academy of Natural Sciences.

Treves, A. (2000). Theory and method in studies of vigilance and aggregation. Animal Behavior, 60, 711-722.

Urbanek, R. P., Szyszkoski, E. K., \& Zimorski, S. E. (2014). Winter distribution dynamics and implications to a reintroduced population of migratory Whooping Cranes. Journal of Fish and Wildlife Management, 5, 340-362.

Wood, C., Qiao, Y., Li, P., Ding, P., Lu, B., \& Xi, Y. (2010). Implications of rice agriculture for wild birds in China. Waterbirds, 33, 30-43. 